\title{
IMPACTOS AMBIENTALES ASOCIADOS A LA CONSTRUCCIÓN DE OBRAS DE CORRECCIÓN HIDROLÓGICA EN LA CUENCA DEL RÍO QUIPAR (SURESTE DE ESPAÑA): APERTURA DE CAMINOS DE ACCESO
}

\author{
F. BELMONTE SERRATO, A. ROMERO DÍAZ y M. MARTÍNEZ LLORIS
}

Departamento de Geografía. Universidad de Murcia. Campus de la Merced. 30001 - Murcia, España.

Correo electrónico de contacto: franbel@um.es

\begin{abstract}
RESUMEN: Se presenta un estudio sobre las consecuencias ambientales derivadas de la construcción de diques de retención de sedimentos en la cuenca del río Quipar. Los caminos de acceso al punto de construcción de los diques tienen un coste ambiental que, en algunos casos, es contrario a la finalidad de dichas construcciones y que difícilmente justifica su construcción dada la escasa capacidad de acumulación de sedimentos del dique en cuestión, o la baja tasa de erosión del barranco donde se construye. El estudio realizado en 6 barrancos de la cuenca del río Quipar, indica que la relación entre el suelo movilizado en los caminos de acceso y el suelo retenido por los diques es de un 10\%, aunque esta oscila entre el $7 \%$ y el 36\%. Por otra parte, la superficie roturada con eliminación de matorral y pinos es de 40 ha en los seis barrancos estudiados (0.7 ha por dique), llegando a 1.6 ha en uno de los barrancos.
\end{abstract}

ABSTRACT: In this paper the environmental consequences derived from the construction of retention sediments dikes in the basin of the Quipar river are studied. The approach roads to the point of construction of the dikes have an environmental cost that, in some cases, is opposite to the purpose of the above mentioned constructions and that doesn't justifies their construction because the scanty capacity of accumulation of sediments of the dike in question, or small rate the erosion of the ravine where it is constructed. The study realized in 6 ravines of the basin of the Quipar river, indicates that the relation between the mobilized soil in the approach roads and the retained soil by the dikes is $10 \%$, though the range of this one is between $7 \%$ and $36 \%$. On the other hand, the plowed surface with bushes and pines elimination is of 40 hectares in the six studied ravines ( 0.7 hectares for dike), being to 1.6 hectares in one of the ravines. 
Palabras clave: Impacto ambiental, caminos de acceso, erosión, diques de corrección hidrológica, SE de España.

Key word: Environmental impact, approach road, erosion, dikes of hydrological correction, south east of Spain.

\section{Introducción}

La construcción de diques de retención de sedimentos, como elementos de corrección hidrológica, en especial en cuencas de gran aporte de sedimentos a los embalses, son unas construcciones bastante usuales en España, y en espacial en el SE peninsular (Romero Díaz, et al, 2004a). No obstante, estos trabajos se hacen especialmente necesarios en zonas semiáridas, debido al grado de degradación de la cubierta vegetal y la torrencialidad de las precipitaciones, que las convierte en áreas gravemente afectas por procesos erosivos. En la cuenca del río Segura, se estima que el número de estas construcciones supera los dos millares. Tan solo dos subcuencas vertientes a los embalse de Puentes y de Alfonso XIII contabilizan mas de 1.300 diques (Sánchez Fuster, 1996).

El objetivo principal de la construcción de los diques trasversales a los cauces, en las cuencas vertientes a los embalses, es controlar y disminuir el aporte de sólidos. Tras su instalación, se estabilizan las laderas, se retienen los acarreos, se disminuye la velocidad del agua, y por tanto su capacidad erosiva, reduciéndose de este modo la cantidad de elementos sólidos que llegan a los embalses, lo cual produce su aterramiento paulatino (Romero Díaz et al, 2004b).

En ocasiones, y debido a la urgente necesidad de frenar o disminuir los efectos sobre los embalses de los procesos erosivos, estos trabajos se realizaron, casi siempre, sin llevar a cabo estudios prospectivos de las consecuencias hidrogeológicas, geomorfológicas o erosivas, que dichos trabajos podrían tener en los cauces y en su entorno inmediato, y, mucho menos, estudios del impacto ambiental generado por los trabajos necesarios para la construcción de dichos diques.

Los primeros trabajos de construcción de diques en la cuenca estudiada, la del río Quipar, se llevaron a cabo por la Confederación Hidrográfica del Segura en 1962. En este proyecto, de corrección hidrológica y forestal se realizan trabajos en 40 ramblas y barrancos, construyendo 349 diques y un gran número de albarradas de piedra en seco.

En fecha tan temprana, los estudios de impacto ambiental no eran obligatorios, por tanto, los trabajos se realizaron sin estudios previos de impacto ambiental.

En 1996 (CHS, 1992), un nuevo proyecto, repara 58 diques, recrece 101 y construye 76 diques nuevos. En este caso, el proyecto correspondiente si va acompañado de un Estudio de Impacto Ambiental, aunque en el mismo se resalta la no obligatoriedad de su 
elaboración, en cuanto a la construcción de los diques, basándose en el Real Decreto Ley $1302 / 86$ de 28 de Junio.

Es evidente que la construcción de estas obras en barrancos, en la mayoría de los casos, de muy difícil acceso, requiere de la previa preparación de caminos que faciliten la introducción de los materiales y maquinaria necesaria hasta el punto elegido, con el consiguiente impacto geomorfológico, erosivo, hidrológico y ecológico. En algunos de los diques más antiguos, no se aprecian caminos construidos expresamente para el acceso, pero en los nuevos diques construidos en 1996 y en los antiguos reparados o recrecidos, los caminos de acceso construidos para el paso de maquinaria pesada hasta el mismo dique (en el propio cauce), adquieren dimensiones considerables.

La visita a todos los diques de esta cuenca ha permitido observar que, en ningún caso, las zonas roturadas para la construcción de estos caminos, han sido de nuevo reforestadas, ni siquiera los tramos de acceso al fondo de los cauces, que en muchas ocasiones tienen pendientes superiores al 100\% (Figura 1) y en algunos casos, encajamientos sobre la superficie original de más de 2 metros (Figura 2).

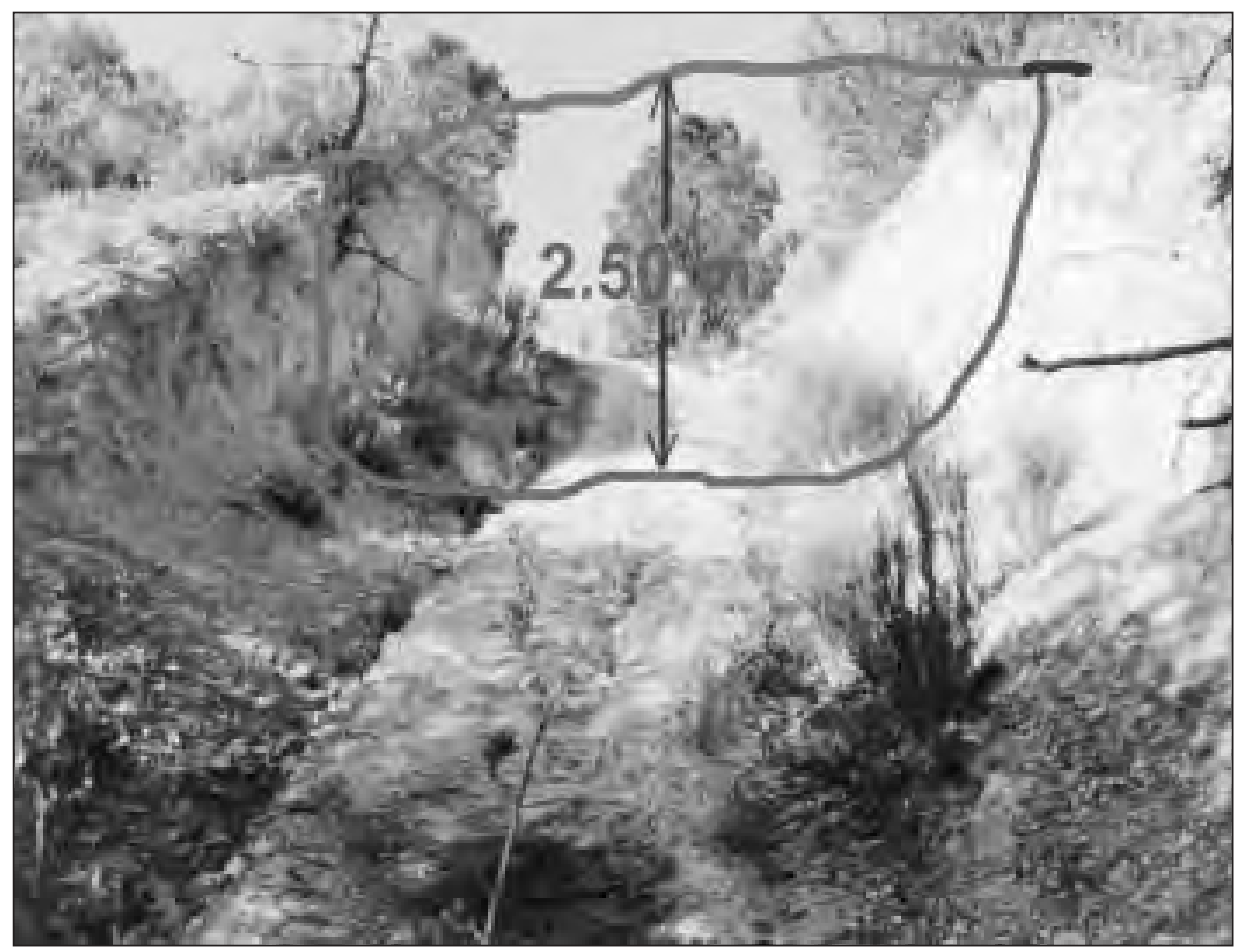

Figura 1. Camino de acceso al cauce desde un campo de cultivo para facilitar el paso de materiales y maquinaria necesarios para la construcción de un dique. 


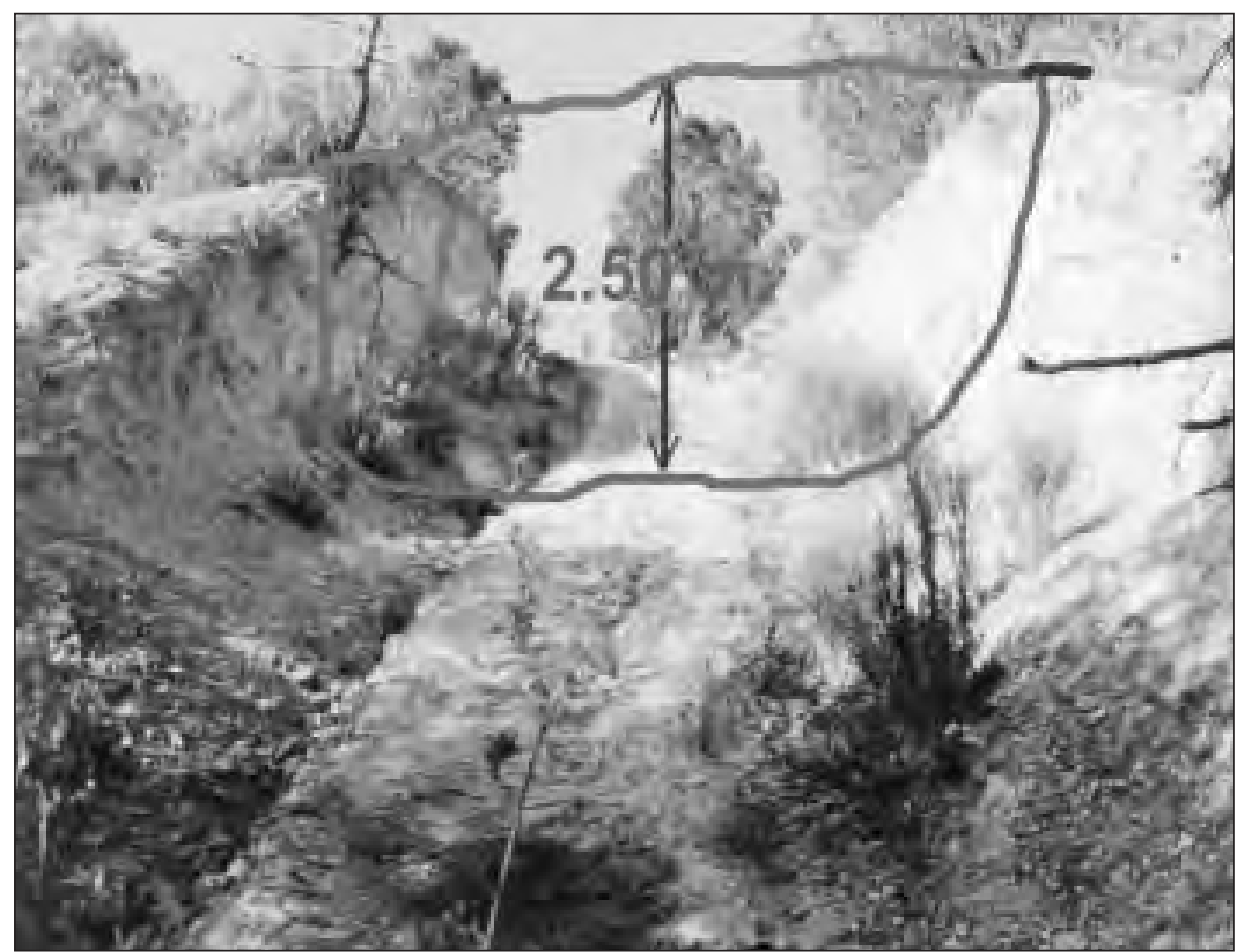

Figura 2. Sección de vaciado sobre el nivel original en un tramo de acceso al cauce para la construcción de un dique.

\section{2. Área de estudio}

La cuenca del río Quipar con una superficie de $826.4 \mathrm{~km}^{2}$, es afluente del Segura por su margen derecha y se sitúa en el centro occidental de la Región de Murcia (Figura 3).

Su altitud va de los 200 a los $1.900 \mathrm{~m}$, si bien, el $50 \%$ de su superficie se sitúa por encima de los $800 \mathrm{~m}$. Las calizas y dolomías dominan la parte alta de la cuenca, mientras que la parte media y baja está caracterizada por una gran variedad litológica en donde aparecen calizas, margas, margocalizas, margas yesíferas, yesos, arenas, areniscas, y formaciones cuaternarias. Los suelos predominantes, desarrollados sobre esas litologías son diferentes tipos de regosoles, leptosoles, calcisoles y fluvisoles.

La vegetación está constituida principalmente por bosques de coníferas xerófilas de Pinus halepensis, y diversos matorrales de distintos porte como: coscojares, enebrares, romerales, garrigas, albaidales, espartizales y tomillares. La protección del suelo por la vegetación suele ser menor en los suelos que más lo necesitan, como son los suelos de 
litología margosa, en los cuales la vegetación suele estar constituida por matorrales de escaso porte y baja densidad.

La precipitación media anual es de $287 \mathrm{~mm}$ en la parte baja de la cuenca y en torno a los $400 \mathrm{~mm}$ en cabecera, con una elevada irregularidad interanual. Las temperaturas medias anuales varían entre los 12 y $\operatorname{los} 16^{\circ} \mathrm{C}$. Según la clasificación de Turc, toda la cuenca posee un clima semiárido.

\section{Metodología}

En este trabajo se han elegido seis barrancos de la subcuenca 6, la más cercana al embalse, y la que posee el mayor número de diques, y se ha medido la longitud, anchura y profundidad de los caminos abiertos expresamente para el acceso en la construcción de los diques, y con esto se ha calculado el volumen de suelo removido y la superficie total roturada, relacionando ambos parámetros con el número de diques y su capacidad de almacenamiento de sedimentos.

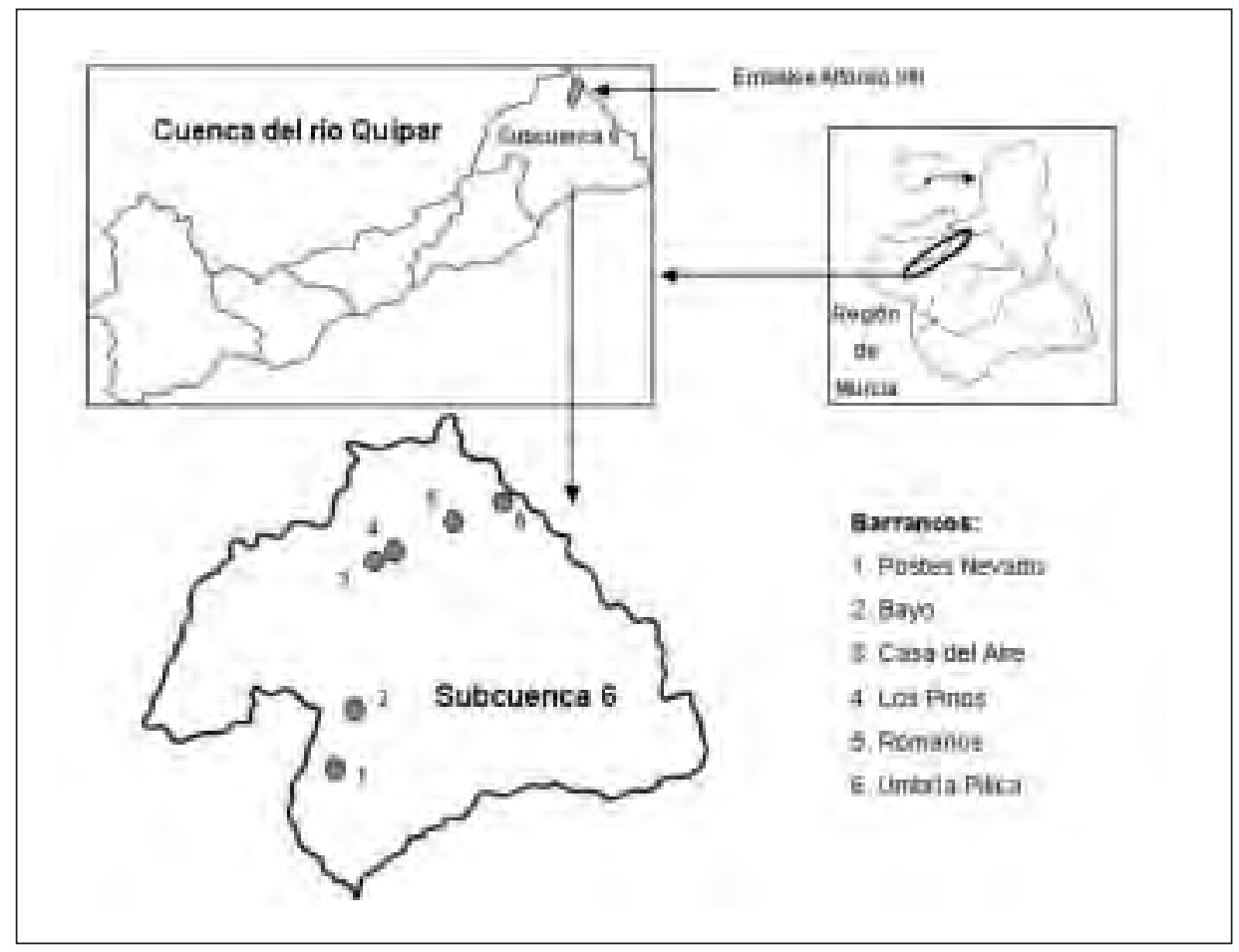

Figura 3. Localización de los barrancos seleccionados 
Sobre el mapa 1: 5.000 se ha medido la longitud, anchura y profundidad de suelo removido, de todos los caminos identificados como construidos expresamente para el acceso a los diques. Luego, sobre el terreno, se han completado tramos que no aparecían en el mapa y se han desechado aquellos tramos que se ha comprobado que ya existían previamente para dar acceso a fincas agrícolas.

Después, se ha medido la superficie de la cuña de sedimentos de cada dique y se ha estimado el volumen de los mismos según la forma geométrica más adecuada para su cálculo (Hernández Laguna et al, 2004). .

Con estas medidas se ha calculado, para cada barranco, la longitud total de caminos, la superficie total roturada y el volumen de suelo movilizado, y esto se ha puesto en relación con el número de diques de cada barranco y con el volumen de sedimentos contenidos en los mismos.

\section{Resultados y discusión}

\section{1 Los caminos}

Casi todos los caminos estudiados se han abierto partiendo de caminos agrícolas próximos o pistas forestales, los tramos de acceso a cada dique suelen ser cortos 200$300 \mathrm{~m}$, aunque en algunos casos, esos tramos superan $1 \mathrm{Km}$. En ocasiones el acceso se ha llevado a cabo a través de un campo de cultivo, por lo que el camino ha quedado eliminado tras la construcción del dique en cuestión. En otros casos, ha sido imposible determinar el lugar por el que se ha accedido para la construcción del dique, probablemente porque se pudo acceder sin necesidad de abrir camino y los cuarenta años transcurridos han borrado las huellas de las rodaduras.

Tabla 1. Longitud, superficie y volumen de suelo movilizado en los caminos abiertos para el acceso a los diques.

\begin{tabular}{|c|c|c|c|c|}
\hline Barrancos & $\begin{array}{c}\text { Long. caminos } \\
(\mathrm{m})\end{array}$ & $\begin{array}{c}\text { Sup. Roturada } \\
\left(\mathrm{m}^{2}\right)\end{array}$ & $\begin{array}{c}\text { Suelo movilizado } \\
\left(\mathrm{m}^{3}\right)\end{array}$ & $\begin{array}{c}\text { Suelo movilizado } \\
(\mathrm{Tm})\end{array}$ \\
\hline 1 & 2350 & 9400 & 2820 & 3384 \\
2 & 1980 & 7920 & 2376 & 2851.2 \\
3 & 1080 & 4320 & 1296 & 1555.2 \\
4 & 500 & 2000 & 600 & 720 \\
5 & 3200 & 12800 & 3840 & 4608 \\
6 & 825 & 3300 & 990 & 1188 \\
Total & 9935 & 39740 & 11922 & 14306.4 \\
\hline
\end{tabular}


La longitud total de caminos abiertos para la construcción de los diques es de unos $10 \mathrm{Km}$. La anchura media de estos caminos es unos $4 \mathrm{~m}$ y la profundidad de suelo movilizado de $30 \mathrm{~cm}$. Con ello, la superficie roturada es de casi 4 hectáreas (tabla1), lo que supone un volumen de suelo movilizado de $12.000 \mathrm{~m}^{3}$, equivalente a unas 14.300 toneladas.

\section{2 Los diques}

En los diques se ha medido la superficie de la cuña de sedimentos, y se ha estimado el volumen de los sedimentos retenidos. Como es lógico, la cantidad de sedimentos retenidos en cada barranco está en relación directa con el número de diques, o lo que es lo mismo con la capacidad de retención de los diques construidos (tabla 2). En el conjunto de los diques estudiados se han retenido, en los 41 años transcurridos desde su construcción, en torno a $120.000 \mathrm{~m}^{3}$ de suelo que viene a corresponder a unas 144.000 toneladas.

El volumen de sedimentos retenidos por dique oscila entre los $2.800 \mathrm{~m}^{3}$ y los 655 $\mathrm{m}^{3}$, situándose la media en algo más de $2.100 \mathrm{~m}^{3}$

Tabla 2. Número de diques construidos y sedimentos acumulados

\begin{tabular}{|c|c|c|c|c|}
\hline Barrancos & $\begin{array}{c}\text { Diques } \\
\text { construidos }\end{array}$ & $\begin{array}{c}\text { Sedimentos } \\
\left(\mathrm{m}^{3}\right)\end{array}$ & $\begin{array}{c}\text { Sedimentos } \\
(\mathrm{Tm})\end{array}$ & $\begin{array}{c}\text { Sed/dique } \\
\left(\mathrm{m}^{3}\right)\end{array}$ \\
\hline 1 & 17 & 41534 & 49841 & 2443.2 \\
2 & 5 & 6651 & 7981 & 1330.2 \\
3 & 7 & 4589 & 5507 & 655.6 \\
4 & 4 & 4044 & 4853 & 1011 \\
5 & 19 & 53222 & 63866 & 2801.1 \\
6 & 5 & 10379 & 12454 & 2076.8 \\
Total & 57 & 120419 & 144503 & 2112.6 \\
\hline
\end{tabular}

\subsection{Coste ambiental}

El objetivo fundamental de este trabajo es valorar algunos de los efectos ambientales de la apertura de caminos para facilitar el acceso a los lugares elegidos para la construcción de los diques de retención de sedimentos y, más concretamente, la longitud, superficie $\mathrm{y}^{3}$ de suelo movilizado por esos caminos.

El resultado de este análisis sugiere que sería imprescindible realizar una valoración previa del coste ambiental de estas obras de corrección hidrológica, al menos, en aquellos lugares que, por sus dificultades de acceso, necesitan la apertura de caminos. 
La longitud media de caminos abiertos para el acceso a la construcción de los diques en los barrancos seleccionados es de $160 \mathrm{~m}$ por dique, que equivale a una superficie roturada de casi $700 \mathrm{~m}^{2}$ (tabla 3) y, aunque no ha sido objeto de este trabajo, en muchos casos esta superficie estaba cubierta por matorral mediterráneo que incluye especies protegidas o estrictamente protegidas por la legislación de la Comunidad Autónoma de Murcia de Orden de 17 de Febrero de 1989, como Pistacia lentiscus (Lentisco), Juniperus oxycedrus, Juniperus comunis (Enebros), Quercus coccifera (coscoja), Rhamnus lycioides (Espino negro), Olea europea Subsp. Sylvestris (Acebuche) Tamaris boveana (Taray) entre otras.

Aunque esta superficie media roturada por dique construido, es bastante homogénea en cinco de los seis barrancos, en el barranco 2 (El Bayo), alcanza los $1600 \mathrm{~m}^{2}$., aproximándose la longitud de camino necesario para cada dique a los $400 \mathrm{~m}$.

Este caso es especialmente significativo, ya que si el objetivo último de la construcción de diques, es disminuir el aterramiento de embalses reteniendo los sedimentos en los barrancos, en este caso, la construcción de los diques no era necesaria ya que desde hace años es un barranco endorreico, que no vierte al río, tras la construcción en su desembocadura de una balsa de lavado de mineral de las minas de Gilico ahora dedicadas a la extracción de áridos.

Tabla 3. Longitud de caminos, superficie roturada, suelo movilizado y porcentaje de suelo movilizado respecto al suelo retenido por los diques.

\begin{tabular}{|c|c|c|c|c|}
\hline Barrancos & $\begin{array}{c}\text { Longitud camino } \\
\text { por dique }(\mathrm{m})\end{array}$ & $\begin{array}{c}\text { Superf. roturada } \\
\text { por dique }\left(\mathrm{m}^{2}\right)\end{array}$ & $\begin{array}{c}\text { Suelo movilizado } \\
\text { por dique }\left(\mathrm{m}^{3}\right)\end{array}$ & $\begin{array}{c}\text { Suelo movilizado/ } \\
\text { Sed. dique (\%) }\end{array}$ \\
\hline 1 & 138.2 & 552.9 & 165.9 & 6.8 \\
2 & 396 & 1584 & 475.2 & 35.7 \\
3 & 154.3 & 617.1 & 185.1 & 28.2 \\
4 & 125 & 500 & 150 & 14.8 \\
5 & 168.4 & 673.7 & 202.1 & 7.2 \\
6 & 165 & 660 & 198 & 9.5 \\
Media & 160.2 & 697.2 & 209.2 & 9.9 \\
\hline
\end{tabular}

En conjunto, la superficie media roturada ha supuesto la movilización de cerca de $210 \mathrm{~m}^{3}$ de suelo por dique construido, lo que supone en torno al $10 \%$ del volumen medio de sedimentos acumulados en los diques.

Pero de nuevo en el barranco 2, los efectos ambientales de la apertura de caminos, adquiere dimensiones importantes, ya que el volumen de suelo movilizado por dique, es superior a un tercio del volumen de suelo retenido en esos diques. En este caso, junto 
con el barranco 3 (Casa del Aire) con un 28\% de suelo movilizado respecto al retenido en los diques y el barranco 4 (Barranco de los Pinos) con un 15\% son especialmente graves.

\section{Conclusiones}

La apertura de caminos de acceso a los puntos seleccionados para la construcción de los diques de retención de sedimentos resulta imprescindible cuando las dificultades de acceso son importantes, pero las consecuencias ambientales pueden resultar demasiado graves para el escaso resultado que en ocasiones tienen determinados diques.

La longitud total de caminos abiertos en los seis barrancos estudiados se aproxima a los $10 \mathrm{Km}$. Esto supone una superficie roturada de más de 4 hectáreas y la movilización de $12.000 \mathrm{~m}^{3}$ de suelo.

El total de sedimentos acumulados en los diques supera los $120.000 \mathrm{~m}^{3}$, que supone una media de algo más de $2100 \mathrm{~m}^{3}$ por dique construido.

Estas cifras indican que se ha necesitado la apertura de una media de $160 \mathrm{~m}$ de camino por dique, lo que supone una superficie roturada de unos $700 \mathrm{~m}^{2}$, aunque, en algún caso, el camino necesario ha sido de casi $400 \mathrm{~m}$ y la superficie roturada de 1600 $\mathrm{m}^{2}$.

Todo esto supone un coste ambiental que sería necesario considerar antes de plantear la apertura de nuevos caminos, ya que el volumen medio de suelo movilizado para la apertura de esos caminos alcanza el $10 \%$ del volumen de suelo retenido en los diques 40 años después de su construcción, llegando a casi el 36\% en el barranco 2 , al $28 \%$ en el 3 y al $15 \%$ en el 4 .

Con todo, lo más grave es que estos caminos quedaron abandonados, tras su construcción, a merced de los procesos erosivos, sin que se llevara a cabo ningún trabajo de restitución vegetal, ni mucho menos, la eliminación física del propio camino.

\section{Agradecimientos}

Este trabajo se ha realizado en el marco del proyecto de investigación REN200203426/HID, financiado por el Ministerio de Ciencia y Tecnología, y fondos FEDER. A ellos nuestro agradecimiento. 


\section{Referencias}

Alonso Sarría, F., Belmonte Serrato, F., Marín SAnleandro, P., Martínez LloRis, M., Ortíz Silla, R., Rodriguez Estrella, T., Romero Díaz, A. y SANCHEZ TORÍBIO, M.I. (2003): La función de los diques de retención de sedimentos y su relación con las características litológicas de sus cuencas. Cuenca del río Quipar, Región de Murcia. En R. Bienes y M.J. Marques (Eds.) Control de la erosión y degradación del suelo. Instituto Madrileño de Investigación Agraria y Alimentaria. Madrid, 527530 .

Hernández Laguna, E., Martínez Lloris, M. y Romero Díaz, A. (2004): Determinación del volumen de sedimentos retenidos en diques de corrección hidrológica. En G. Benito \& A. Díez Herrero (Eds.), Riesgos Naturales y Antrópicos en Geomorfología. SEG y CSIC, Madrid, 201-210.

Martínez Lloris, M., Romero DíAZ, A. y Alonso SARRÍA, F. (2001): Respuesta erosiva de cuencas, corregidas mediante diques de retención de sedimentos, ante lluvias de alta intensidad. Cuenca del río Quipar, Sureste de España. Papeles de Geografía, 34: 191-203.

Romero díaz, A., Martínez Lloris, M., Belmonte Serrato, F. y Hernández LAGUNA, E. (2004a): . La cuenca del río Quipar (Región de Murcia): una cuenca modelo para el estudio de utilidad de los diques de retención de sedimentos en el control de la erosión y en la recarga de acuíferos. Nimbus , 11-12: 89-105

Romero Díaz, A., Martínez Lloris, M., y Belmonte Serrato, F. (2004b): The construction of dikes of hydrological correction as policy to retain the erosion and to avoid the silting up of dams in the Segura basin (Spain). En A. Faz, R, Ortiz \& G. García (Eds), Fourth International Conference on Land Degradation. Cartagena, Topic 7 (23). Publicado en Cd.

SÁNCHEZ Fuster, M.C. (1996): Actions des organismes publics tenant de freiner la perte de sol dans le bassin du Segura, Murcia (Espagne). En F. López Bermúdez y P. Gognon (Eds.) Erosion hydrique, desertification et aménagement dans l'environnement Méditerranéen semi-aride. Universidad de Murcia, 235-247. 